\title{
Hacia la validación del SUSESO ISTAS 21 versión breve en trabajadores de hospitales públicos
}

\section{Towards the validation of the SUSESO ISTAS 21 short version in workers of public hospitals}

\author{
Rodolfo Mendoza-Llanos \\ Universidad del Bío-Bío \\ Emilio Moyano-Díaz \\ Universidad de Talca
}

Recibido (22 de mayo de 2017) Aceptado (28 de febrero de 2019)

\begin{abstract}
Resumen
La mitad de la población mundial son trabajadores, por lo que invertir en políticas de salud hacia ellos es muy relevante. La Organización Mundial de la Salud (OMS) insta a los estados miembros a generar planes de salud ocupacional evaluables para promover el bienestar de los trabajadores, para lo cual se requiere contar con instrumentos adecuados. Se estudian aquí propiedades psicométricas del instrumento para evaluar riesgo psicosocial SUSESO-ISTAS 21 versión breve (20 ítems), aplicado a 341 trabajadores hospitalarios (región del Maule, Chile), conjuntamente con la DASS21 (Depresión, Ansiedad y Estrés). La confiabilidad general es adecuada, reafirmando informes previos. Respecto de validez, cuatro de las cinco dimensiones de la versión breve no alcanzan valores psicométricos adecuados, no confirmándose la estructura factorial teorizada. Se obtiene apoyo a la validez convergente con DASS21. Se discute y propone acerca de lo psicométrico y sus usos eventuales.

Palabras clave: Riesgo psicosocial, salud ocupacional, confiabilidad, SUSESO ISTAS 21
\end{abstract}

\begin{abstract}
Workers represent half of the world's population, so investing in public health policies aimed at them has enormous importance. The World Health Organization (wHO) urges member states to generate assessable occupational health plans that promote their wellbeing. The study aims to evaluate the psychometric properties of ISTAS-SUSESO 21 short version (20 items), which is an instrument for assessing psychosocial risk developed for Chile. We performed reliability analysis, confirmatory factor analysis using structural equation model and convergent validity analysis with the DASS21 Scale (Depression, Anxiety and Stress) using a sample of 341 hospital employees in the region of Maule (Chile). Overall reliability is adequate, reaffirming previous reports. Regarding validity, four of five dimensions of the short version does not reach adequate psychometric values, and theorized factorial structure is not confirmed. We found support for convergent validity with DASS 21, and discussed the psychometric and its possible uses.

Keywords: Risk factors, occupational health, reliability and validity
\end{abstract}

Agradecimientos: el primer autor a la Universidad de Talca por la Beca de Doctorado otorgada.

Correspondencia: Emilio Moyano Díaz, Grupo de Investigación en Calidad de Vida y Ambientes Saludables (GICVAS), Facultad de Psicología, Universidad de Talca (Chile). emoyano@utalca.cl 


\section{Introducción}

El Plan de Acción Mundial sobre la Salud de los Trabajadores informa que éstos son la mitad de la población del mundo y los máximos contribuyentes al desarrollo económico y global (OMS, 2010). La Organización Mundial de la Salud (OMS) calculó que anualmente se producían 160 millones de nuevos casos de enfermedades relacionadas con el trabajo (OMS, 2010), y en 2015 la Organización Internacional del Trabajo (OIT, 2015) informó que anualmente 2.3 millones de trabajadores mueren por accidentes o enfermedades laborales. Así, no cabe duda que ocuparse de esto constituye una tarea desafiante y necesaria.

El plan 2015-2025 de la OPS (2015) consiste en un seguimiento al "Plan de acción mundial sobre la salud de los trabajadores 2008 - 2017" y mantiene la iniciativa de la OMS (2010) de instar a los estados miembros a asegurar una acción concertada entre sus programas nacionales de salud para los trabajadores, respecto de la prevención de riesgos de enfermedades crónicas, promoción sanitaria, salud ambiental y mental. Se busca que los trabajadores puedan disfrutar del más alto nivel posible de salud física y mental, de condiciones de trabajo favorables, y reducir las desigualdades de salud. Su meta es "fortalecer la respuesta del sector de la salud, en coordinación con los demás sectores involucrados, para brindar atención integral a la salud de los trabajadores, mejorar los entornos de trabajo, aumentar los esfuerzos para promover la salud de los trabajadores y disminuir las desigualdades en salud mediante la ejecución de políticas, planes y normas actualizados" (OPS, 2015, p.7). El plan contiene cinco líneas de estratégicas de acción, de las cuales la segunda -y especialmente la quinta- ocupan nuestra atención aquí: identificar, evaluar, prevenir y controlar las condiciones y exposiciones peligrosas en el trabajo $\mathrm{y}$, fortalecer la capacidad de diagnóstico, los sistemas de información, la vigilancia epidemiológica y la investigación de las enfermedades, los accidentes y las muertes en el trabajo (OPS, 2015).

La OMS (2010, p.11) ha definido 'entorno de trabajo saludable' a 'aquel en el que los trabajadores y el personal superior colaboran en el desarrollo de procesos de mejora continua para proteger y promover la salud, la seguridad, el bienestar de todos los trabajadores, y la sostenibilidad del lugar de trabajo". Propone un modelo de trabajo con ambientes saludables conformado por cuatro áreas principales, y sobre las que se puede influir: ambiente físico del trabajo; recursos personales de salud; participación de la empresa en la comunidad y ambiente psicosocial del trabajo. Esta última incluye la organización del trabajo y la cultura institucional, actitudes, valores, creencias y prácticas que se observan a diario en la empresa y que afectan el bienestar mental y físico de los empleados.
Según Roman (2006) todos los problemas de salud mental, incluidas las alteraciones psiquiátricas comunes, están relacionados estrecha y frecuentemente en su origen o en su curso, con el trabajo que las personas realizan. La prevalencia de trastornos mentales en Chile ha disminuido de un 36\% en 2004 (Minoletti \& Zaccaria, 2005), a 21\% una década después (Garrido et al, 2013). La progresiva aplicación de la ley 16.744 que hace obligatorio en las empresas el diagnóstico de riesgos psicosociales, sin duda tendrá un impacto positivo en prevención primaria de salud mental en el país. Diversos estudios reportan que los problemas de salud mental son causa de discapacidad y una explicación del ausentismo y de la disminución del rendimiento laboral, por sobre otras patologías (Minoletti \& Zaccaria, 2005; Garrido et al., 2013; Mendoza-Llanos, 2015).

Las políticas públicas nacionales relativas a salud responden a las indicaciones de organismos internacionales referidas a las responsabilidades relacionadas con salud en el trabajo, pero no se observa una especificidad respecto de la salud mental laboral. No obstante, promover la salud mental ocupacional es una estrategia de intervención importante para fomentar la salud de los trabajadores y, por tanto, de la población. Las condiciones laborales son una exposición crónica a situaciones de riesgo eventual que no son problemas particulares y que escapan al control individual, constituyéndose en un verdadero problema de salud pública cuando su tasa de prevalencia e incidencia resulta alta (Gil-Monte, 2012).

En este contexto, se ha definido los factores psicosociales como aquellas condiciones presentes en situaciones laborales -organización, tareas y diseño del lugar de trabajo, salario, estabilidad, etc.- que pueden afectar el desarrollo y la salud de quienes realizan el trabajo, y que pueden conducir a estrés (Gil-Monte, 2012; Gómez, Hernández \& Méndez, 2014; Ansoleaga, 2015). Los factores psicosociales pueden ser entendidos como factores estresantes del trabajo en general, potencialmente provocadores de estrés emocional o mental a los trabajadores (OMS, 2010), y una forma de medirles y evaluarles en Chile, es por medio de medidas indirectas de estrés de origen laboral (Alvarado et al., 2012). Así, se requiere avanzar para disponer con algún instrumento que permita medir confiable y válidamente aquellos factores de modo directo y específico (Gil-Monte, 2012; Ansoleaga, 2015). Por cierto, los factores psicosociales también pueden favorecer la actividad laboral, facilitando o promoviendo el desarrollo personal, la salud y el bienestar de los trabajadores.

La entidad gubernamental chilena 'Superintendencia de Seguridad Social' (SUSESO) adaptó una versión española del Cuestionario para evaluar Riesgos Psicosociales ISTAS 21 (Moncada, Llorens, Navarro \& Kristensen, 2005), creado en Dinamarca como CoPSoQ (Copenhagen 
Psychosocial Questionaire, de Kristensen, Hannerz, Høgh $\&$ Borg, 2005). Su manual de uso en Chile informa que se cuenta con dos versiones: un SUSESO ISTAS 21-Versión Completa de 91 ítems, diseñado para ser aplicado principalmente como herramienta de investigación, y una Versión Breve, compuesta de 20 ítems, con las mismas dimensiones de la versión completa, y ambas diseñadas para ser aplicadas con fines de prevención, fiscalización o vigilancia epidemiológica en cualquier institución adscrita al sistema de seguridad social regido por la Ley 16.744 (Saavedra, Fuentealba \& Perez, 2009).

El instrumento SUSESO ISTAS 21 está conformado por cinco dimensiones que son: exigencias psicológicas -carga de trabajo de tipo cuantitativas, cognitivas, emocionales, esconder emociones y sensoriales-; trabajo y desarrollo de habilidades -influencia en el trabajo, posibilidades de desarrollo, control de tiempo en el trabajo, sentido del trabajo, integración en la empresa-; apoyo social en la empresa y calidad del liderazgo -previsibilidad, claridad de rol, conflicto de rol, calidad de liderazgo, apoyo social de compañeros, apoyo social de superiores, posibilidades de relación social, sentimiento de grupo-; compensaciones -inseguridad y estima- y doble presencia, ésta última buscando incorporar una perspectiva de género referida a las exigencias por las tareas en el trabajo y en el hogar que se producen al mismo tiempo, y que aparecen más frecuentemente en mujeres (Alvarado et al., 2009).

El manual afirma que ambas versiones - de 91 y 20 ítems- serían "equivalentes y capaces de discriminar de igual forma el riesgo" (Saavedra et al., 2009, p. 22), y se reporta que sus propiedades psicométricas fueron evaluadas mediante los siguientes análisis: i) heterogeneidad de los ítems y su correlación con la dimensión correspondiente, ii) correlación de los puntajes de las dimensiones de la versión corta con los puntajes para las mismas dimensiones de la versión completa, iii) convergencia, basado en la correlación de los puntajes de cada dimensión con las puntuaciones en el Cuestionario de Salud General (GHQ - 12) y en el Cuestionario de Salud SF - 36 y, iv) correlación test-re-test (Alvarado et al., 2012). Con anterioridad se ha reportado la existencia de una versión de 72 ítems -intermedia-, que también incluye las cinco dimensiones descritas para las otras versiones (Alvarado et al., 2009)

En la literatura científica se observa que la evaluación de riesgos psicosociales ha sido realizada con diferentes instrumentos (Ansoleaga, 2015; Ceballos, Paravic-Klijn, Burgos \& Barriga, 2014; Cornejo, 2009), y respecto del SUSESO ISTAS 21 de interés aquí, y desde el punto de vista psicométrico, sólo es posible encontrar reportes de confiabilidad y validez para las versiones extensa -91 ítems(Alvarado et al., 2012) e intermedia -72 ítems- (Alvarado et al, 2009) del instrumento. En lo mejor de nuestro cono- cimiento, existen sólo tres estudios publicados en que se ha utilizado la versión breve -20 ítems- del SUSESO ISTAS 21 , los cuales tuvieron por objeto evaluar riesgos psicosociales en muestras de trabajadores de diferentes rubros y no analizar psicométricamente el instrumento. Uno relativo a trabajadores del rubro energético (Güilgüiruca, Meza, Góngora \& Moya, 2015), otro a conductores de locomoción colectiva (Bravo \& Nazar, 2015), y otro a enfermeros de unidades críticas de hospitales (Ceballos-Vásquez et al., 2015). En dos de ellos ha sido reportada la confiabilidad general del instrumento: $\alpha_{\text {global }}=0,769$ (Güilgüiruca, Meza, Góngora \& Moya, 2015) y $\alpha_{\text {global }}=0,62$ (Bravo \& Nazar, 2015), sin reportarse en ninguno de los tres estudios algún análisis relativo a su validez.

El Protocolo de Vigilancia indica que el SUSESO ISTAS 21 breve sea aplicado al inicio de una evaluación de riesgos psicosociales (MINSAL, 2013) y así se está aplicando actualmente en el país. En el manual de uso de la prueba se señala que las propiedades psicométricas de la versión breve serían equivalentes a las de la versión extensa (Saavedra et al., 2009, p. 22) aunque sin entregarse propiamente evidencia de ello. También ha sido sugerido que la versión breve serviría sólo para sensibilizar, o para la evaluación de riesgos en empresas pequeñas (Alvarado et al, 2009). Una revisión de la literatura al respecto muestra, sin embargo, que no se cuenta con ninguna evidencia psicométrica de la equivalencia sugerida entre la versión breve -en uso- respecto de las versiones extensas del instrumento, ni tampoco -como ya se indicó- publicaciones acerca de la validez del ISTAS versión breve. Este instrumento cuenta sin embargo con índices psicométricos de confiabilidad general adecuados, pero se requiere avanzar en aportar evidencia respecto de su validez.

Entendemos que la validación de un instrumento psicométrico es una tarea prolongada, que idealmente requiere muestras amplias y representativas de diferentes rubros ocupacionales en este caso (dado las características de lo que se busca medir) o, en su defecto, numerosos estudios en diferentes muestras ocupacionales. El propósito aquí va en ésta última dirección, aportar preliminarmente a la validez del instrumento SUSESO ISTAS 21 breve, y particularmente a su validez de constructo, mediante su aplicación a una muestra de trabajadores de la salud pertenecientes a diferentes estamentos de hospitales públicos de la región del Maule. Se busca analizar la validez de constructo del instrumento verificando la estructura pentadimensional del instrumento y, también, poniéndole en relación con medidas de depresión, ansiedad y estrés (validez convergente). 


\section{Método}

Una muestra no probabilística de 341 funcionarios de dos hospitales de la región del Maule (71.3\% mujeres) de todos los estamentos (12.8\% auxiliar; $16.9 \%$ administrativo; $34.7 \%$ técnico; $28.2 \%$ profesional no médico y $7.6 \%$ ley médica) respondió voluntariamente el SUSESO-ISTAS21 en su versión breve ya descrita de 20 ítems, y la escala de Depresión, Ansiedad y Estrés (DASS21) (Antúnez \& Vinet, 2012), después de aceptar una invitación al efecto, y de firmar un consentimiento informado. Ambos instrumentos fueron auto-aplicados en grupos de funcionarios por un equipo de profesionales a cargo de la actividad, quienes informaron de los objetivos del estudio. Respecto del cálculo de las propiedades psicométricas del instrumento se procedió mediante la realización de los tres análisis siguientes: para confiabilidad análisis de consistencia interna general y por dimensiones y para aportar a la validez, análisis factorial confirmatorio y de validez convergente.

El análisis de consistencia interna realizado para cada dimensión fue realizado mediante el cálculo de alfas de Cronbach y sus intervalos de confianza. Siguiendo las recomendaciones de algunos autores (Domínguez-Lara, Merino-Soto \& Navarro-Loli, 2016; Domínguez-Lara \& Merino-Soto, 2015) el cálculo de intervalos de confianza permite estimar con mayor precisión el rango probable de valores a obtener por un indicador. Se espera que los valores alfa estimados sean iguales o superiores a 0.7 (DomínguezLara \& Merino-Soto, 2015). Dado que el ISTAS 21 versión breve está conformado por escalas de tipo ordinal se realizará también análisis para obtener el coeficiente Omega de McDonald siguiendo recomendaciones de Elosua \& Zumbo (2008).

El análisis factorial confirmatorio es un medio para construir y evaluar modelos teóricos (Lloret-Segura et al, 2014; Ximénez \& García, 2005) y, así, permite avanzar en la validez de constructo. En esta investigación se realiza un análisis factorial confirmatorio del modelo de cinco dimensiones correspondiente a la estructura teórica del instrumento, por medio de ecuaciones estructurales con método de estimación de máxima verosimilitud, dada su capacidad para recuperar factores débiles en muestras superiores a los 300 sujetos (Ximénez \& García, 2005). La bondad de ajuste del modelo fue evaluada mediante el Índice de Ajuste Comparativo (CFI), el Índice de TuckerLewis (TLI) y el error cuadrático medio de aproximación (RMSEA). Para considerar que un modelo es aceptable se esperan valores de CFI y TLI superiores a 0.90 y RMSEA entre 0.05 y 0.08 (Schermelleh-Engel, Moosbrugger \& Müller, 2003). El modelo de medida se basa en la definición del instrumento según el cual, el factor 1, Exigencias psicológicas, está constituido por los ítems del 1 al 5; el factor dos, Trabajo activo y desarrollo de habilidades, por los reactivos 6 al 10; el factor 3, Apoyo social en empresa y calidad del Liderazgo, conformado por los ítems 11 al 15; el factor cuarto, Compensaciones, por los reactivos 16 al 18, y el quinto factor, Doble Presencia, por el 19 y 20.

El análisis de validez convergente consiste en el uso de la correlación del ISTAS 21 breve con cada dimensión del instrumento DASS-21 utilizada como criterio: depresión, ansiedad y estrés (Antúnez \& Vinet, 2012). Puesto que el DASS-21 recoge elementos de malestar en general respecto de depresión, ansiedad y estrés, y el ISTAS-SUSESO es un instrumento que captura el riesgo en distintas variables psicosociales en el trabajo, se espera que cada dimensión de éste correlacione positivamente con cada dimensión del DASS-21. Para los análisis se utilizó AMOS v21, SPSS v21 y JASP v8.1.

Adicionalmente, y a propósitos no psicométricos sino de información diagnóstica, se reporta los niveles de riesgo obtenidos para los hospitales del presente estudio. Se sigue el criterio del Protocolo de Vigilancia de Riesgos Psicosociales en el Trabajo que establece que cuando más del $50 \%$ de los trabajadores obtienen un puntaje de "riesgo alto" se considera que la dimensión correspondiente está "en riesgo" (MINSAL, 2013, p. 19).

\section{Resultados}

El análisis de confiabilidad mediante consistencia interna del SUSESO-ISTAS21 versión breve mostró un alfa global de valor 0.758 (IC al 95\%: 0.719-0.794) considerado adecuado.

El análisis por dimensiones -mediante alfa de Cronbach y Omega de McDonald- muestra que solo la dimensión Apoyo social en la empresa y calidad del liderazgo de $\alpha=$ 0.71 (Tabla 1 ), alcanza el valor o criterio mínimo esperado $(\alpha \geq 0.7)$.

La búsqueda de la validez de constructo fue realizada por medio de un análisis factorial confirmatorio, utilizando ecuaciones estructurales, para verificar la estructura penta-dimensional del instrumento. Los indicadores obtenidos $-\mathrm{CFI}=0.795 ; \mathrm{TLI}=0.762 ; \mathrm{RMSEA}=0,08$ - dan cuenta de un grado de ajuste inadecuado e inadmisible según los criterios establecidos para aquellos (Schermelleh-Engel, Moosbrugger \& Müller, 2003). Adicionalmente, las correlaciones entre las dimensiones Apoyo social en la empresa y calidad del liderazgo, y Compensaciones, son mayores a 1 en un modelo estandarizado (figura 1), lo que sugiere que podría tratarse no de dos sino y sólo de una dimensión. De este modo, la estructura relativa a las dimensiones del instrumento no solo no emerge adecuadamente, sino que se aleja considerablemente de la configuración pentadimensional del instrumento original. 
Tabla 1. Confiabilidad para las dimensiones de riesgo psicosocial medidas por la SUSESO ISTAS versión breve. $\mathrm{N}=341$

\begin{tabular}{lccc}
\hline Dimensiones & $\alpha$ de Cronbach & IC [95\%] & $\omega$ de McDonald \\
\hline Exigencias Psicológicas & 0.530 & {$[0.449-0.602]$} & 0.579 \\
Trabajo activo y desarrollo de habilidades & 0.529 & {$[0.448-0.601]$} & 0.616 \\
Apoyo social en la empresa y calidad del liderazgo & 0.710 & {$[0.653-0.759]$} & 0.720 \\
Compensaciones & 0.441 & {$[0.351-0.523]$} & 0.521 \\
Doble presencia & 0.408 & {$[0.315-0.493]$} & 0.411 \\
\hline
\end{tabular}

Figura 1. Modelo estandarizado de cinco dimensiones del SUSESO ISTAS 21 versión breve.

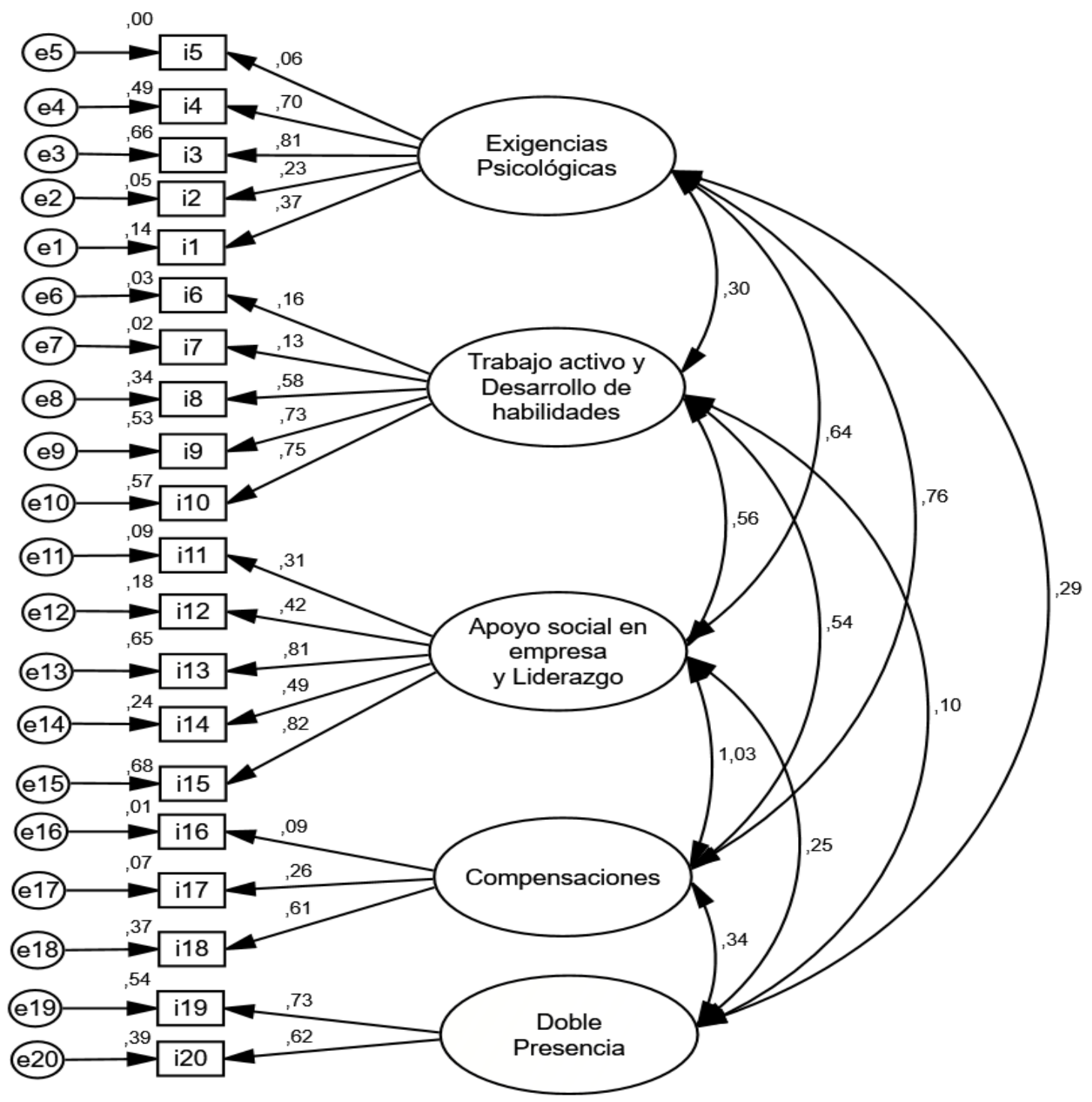


El análisis de validez convergente sin embargo muestra que todas las correlaciones entre ISTAS 21 breve y DASS 21 son estadísticamente significativas y positivas, indicando convergencia entre riesgo psicosocial en el trabajo y malestar -depresión, ansiedad, estrés- lo cual apoya la hipótesis establecida a priori en este estudio (tabla 2). De este modo, si bien la validez relativa a la estructura del instrumento no es la esperada, el significado global de su contenido o ítems parece corresponder a una misma categoría conceptual -concepto de riesgos psicosociales-, al manifestarse en la misma dirección respecto a las variables criterio -depresión, ansiedad y estrés- del DASS 21.

Tabla 2. Correlaciones de la Escala SUSESO-ISTAS 21 versión breve y el DASS21

\begin{tabular}{llllll}
\hline Dimensiones* & EP & TD & AL & Co & DP \\
\hline Depresión & $.170^{* *}$ & $.212^{* *}$ & $.318^{* *}$ & $.227^{* *}$ & $.279^{* *}$ \\
Ansiedad & $.159^{* *}$ & $.170^{* *}$ & $.251^{* *}$ & $.244^{* *}$ & $.283^{* *}$ \\
Estrés & $.210^{* *}$ & $.171^{* *}$ & $.300^{* *}$ & $.288^{* *}$ & $.282^{* *}$ \\
\hline
\end{tabular}

Nota. $* * \mathrm{p}<0.01$.

*EP = Exigencias Psicológicas; $\mathrm{TD}=$ Trabajo activo y desarrollo de habilidades; $\mathrm{AL}$ = Apoyo social en empresa y calidad del Liderazgo;

$\mathrm{Co}=$ Compensaciones; $\mathrm{DP}=$ Doble Presencia

Finalmente, y solo a propósitos de ilustración diagnóstica, reportamos los resultados correspondientes a riesgos psicosociales obtenidos del análisis de los datos relativos a trabajadores hospitalarios de esta muestra.
Descriptivamente, los datos muestran que para dos de las cinco dimensiones se obtuvo 'alto riesgo', tratándose de las dimensiones Compensaciones y Doble presencia (Tabla 3).

Tabla 3. Riesgo psicosocial por dimensión del SUSESO-ISTAS 21: frecuencia y porcentaje para trabajadores de la salud de la Región del Maule $(N=341)$.

\begin{tabular}{lcccccc}
\hline \multirow{2}{*}{ Dimensiones SUSESO-ISTAS 21 } & \multicolumn{2}{c}{ Nivel bajo } & \multicolumn{2}{c}{ Nivel medio } & \multicolumn{2}{c}{ Nivel alto } \\
\cline { 2 - 7 } & $\mathrm{n}$ & $\%$ & $\mathrm{n}$ & $\%$ & $\mathrm{n}$ & $\%$ \\
\hline Exigencias Psicológicas & 207 & 61 & 99 & 29 & 35 & 10 \\
Trabajo activo y desarrollo de habilidades & 126 & 37 & 127 & 37 & 88 & 26 \\
Apoyo social en la empresa y calidad del liderazgo & 49 & 14 & 146 & 43 & 146 & 43 \\
Compensaciones & 21 & 6 & 52 & 15 & 268 & 79 \\
Doble presencia & 36 & 11 & 85 & 25 & 220 & 65 \\
\hline
\end{tabular}




\section{Discusión}

Contar con instrumentos para medir riesgos psicosociales en los ambientes laborales es de primera importancia si se quiere detener, e idealmente disminuir, los trastornos mentales en la población de un país. Es una estrategia conveniente económicamente, toda vez que no descansa su costo de aplicación principalmente en el aparato público, sino que se le hace dependiente y viable mediante instituciones privadas. Para ello, contar con instrumentos convenientemente probados, con adecuadas propiedades psicométricas, es de primera importancia. Por cierto, alcanzar óptimas propiedades psicométricas es una tarea de largo plazo, muy especialmente cuando se trata de medidas de fenómenos complejos y de relevancia en sus eventuales consecuencias. En el presente estudio se propuso avanzar sumando análisis y resultados para ir dando cuenta de las propiedades psicométricas de la versión breve del SUSESO ISTAS 21 y particularmente de su validez, ya que se carece de reportes al respecto para esta versión. Se trata de un instrumento que además de confiable globalmente, es económico de aplicación, y promisorio, pero acerca del cual se desconoce su validez para poblaciones chilenas.

Esta versión breve presenta propiedades psicométricas con índices de confiabilidad generales $-\alpha$ de Cronbach, $\omega$ de McDonald e intervalos de confianza- similares a los previamente reportados para muestras de trabajadores chilenos. En la presente aplicación a trabajadores hospitalarios públicos de diferentes estamentos se alcanzó un alfa de Cronbach global de 0.758, adecuado, que se sitúa entre los valores reportados previamente: 0.769 de Güilgüiruca, et al. (2015) y 0.62 reportado por Bravo \& Nazar (2015).

El análisis de confiabilidad específico para cada dimensión muestra sin embargo que solo una dimensión del instrumento obtuvo un valor aceptable superior a 0.7 y corresponde a la dimensión Apoyo social en la empresa y calidad del liderazgo $(\alpha=0.71)$. No se cuenta con reportes previos al respecto que pudieran servir de comparación para ponderar este resultado, sin perjuicio de lo cual una primera interpretación - ortodoxa - es que se trata de una debilidad ilustrativa de que las dimensiones aparecen con poca homogeneidad interna, o heterogéneas respecto del aspecto del constructo riesgo psicosocial, que el instrumento busca medir.

Respecto de su validez, de particular interés o foco en el presente estudio, y específicamente de su validez de constructo, observamos que no se ha re-encontrado aquí, para esta muestra de trabajadores de hospitales públicos, la estructura pentadimensional del instrumento original al aplicar análisis factorial confirmatorio a los resultados. Las altas correlaciones entre dos de sus dimensiones reflejan una superposición o falta de diferenciación de sus ítems como componentes de dos dimensiones que se quiere distintas, las cuales no aparecen claramente diferenciadas como se espera, de modo que pudieran no estar recogiendo empíricamente, y para los fines diagnósticos pertinentes, lo que se busca diferenciar.

Sin embargo, se obtuvo un resultado positivo relativo a validez convergente, al encontrarse correlaciones positivas del instrumento con medidas de depresión, ansiedad y estrés. Esto da apoyo indirecto general a la validez de constructo de la escala.

Sin perjuicio de lo anterior, es de destacar también que convergentemente con reportes de estudios previos para con versiones más extensas, la dimensión denominada 'doble presencia' tiene muy escasa confiabilidad aquí, motivo por lo cual su inclusión como parte del instrumento debe ser revisada, y ser mejorada de considerarse necesaria su mantención. Formas de mejorar esta dimensión incluyen — por ejemplo- que se le agregue o, sustituya algunos de sus ítems para probarles en nuevos estudios psicométricos, como ha sido sugerido previamente (Alvarado et al, 2009), lo cual permanece como desafío hasta hoy. Por cierto, una discusión de mayor alcance es aquella relativa a la decisión de mantener o no esta dimensión como parte del instrumento. A nuestro modo de ver, y en un nivel teórico, más allá del conflicto demanda-control que pudiera verse reflejado en la doble presencia, ésta última pudiera interpretarse como más propia del ámbito del conflicto trabajo-familia que a riesgo psicosocial en el trabajo, por lo que su evaluación como causa potencial de estrés con ocasión del trabajo es cuestionable, aunque pueda ser un área de intervención valorable en la gestión de personas, incluso como parte de la responsabilidad social de la empresa u organización. Entendemos que algunas características del trabajo en esta muestra en particular — por ejemplo, el sistema de turnospudiesen considerarse elementos desencadenantes de doble presencia, pero aun así, una persona podría experimentar la denominada doble presencia al estar alejado de su hogar en actividades tan distintas al trabajo como las relativas a vacaciones u ocio.

Más globalmente, dados los resultados psicométricos encontrados, la versión breve del instrumento debiera ser utilizada profesionalmente con cautela, ya que su confiabilidad por dimensión es baja. Al no existir estudios internacionales con una versión ISTAS Breve igual a la breve chilena no es posible - strictu sensu- realizar comparaciones de resultados entre mismas versiones. Así, la versión breve inicial española tiene 38 ítems distribuidos en 6 dimensiones (ISTAS, 2003) mientras que la más reciente versión CoPsoQ II en su versión breve- está conformada por 28 ítems en 14 dimensiones (Moncada et al., 2014). A su vez la versión breve danesa tiene 44 ítems en 8 dimensiones (Kristensen et al, 2005). En resumen, todas estas son 
versiones breves, pero más extensas que la versión breve chilena, y aparecen con indicadores de confiabilidad mejores que la local. Así podría también pensarse en la adopción de alguna de aquellas o en un nueva versión local.

Sin perjuicio de lo anterior y, con base en los resultados aquí encontrados y las referencias previas analizadas, si se persistiera en el uso profesional de la versión breve del SUSESO-ISTAS21 parece más apropiado hacerlo junto con algún otro instrumento(s) complementario(s) (al menos en muestras similares a la de este estudio) y, aunque no ha sido sugerido su uso para propósitos de investigación de los riesgos psicosociales en el trabajo, existen reportes para estos fines (Güilgüiruca et al, 2015; Ceballos-Vásquez et al, 2015, Bravo \& Nazar, 2015). Por cierto, ella requiere más estudios psicométricos para eventuales correcciones o mejoramientos de su confiabilidad por dimensiones $\mathrm{y}$, muy especialmente, para contribuir a reunir evidencia acerca de su validez dada la carencia de estudios al respecto. Considerando las debilidades reportadas en este análisis de validez preliminar, su utilización como instrumento de screening con objetivos preventivos incluyendo sus cinco dimensiones — que es lo sugerido en el manual—, podría ser errada. Así, debiera usarse - toda vez que ello sea posible- las versiones más extensas del SUSESO-ISTAS de 72 y 91 ítems siempre que se quiera realizar una fiscalización o vigilancia epidemiológica propiamente tales. Esto implica un costo un poco mayor en cuanto a tiempos de aplicación (lo que creemos es compensado extensamente) pero permite determinar con precisión confiable las áreas de salud ocupacional eventualmente más afectadas $\mathrm{y}$, por tanto, servir de guía segura respecto de qué factores psicosociales se debiera intervenir.

Evaluar riesgos psicosociales en las organizaciones de trabajo en general — públicas y especialmente privadas que son la mayoría - puede ser considerado un medio de prevención primaria muy valioso que cobra aún mayor relevancia y utilidad dadas las históricas restricciones presupuestarias en el ámbito de la salud mental desde el aparato público (Minoletti \& Zaccaria, 2005; Valdés \& Errázuriz, 2012). Pero aplicar versiones más extensas del SUSESO ISTAS como se realiza en países más desarrollados (Dinamarca especialmente, y también España) no es significativamente más caro que aplicar la actual aplicación de la versión breve. Especialmente, si se quiere evitar 'falsos negativos' que tienen consecuencias sobre la salud mental y así, económicos. En Chile la tasa global de trastornos mentales ha disminuido lo cual es muy positivo, pero respecto del suicidio ha aumentado muy fuertemente (Guajardo, Ojeda, Achui \& Larraguibel, 2015; MoyanoDiaz \& Barría, 2006), de modo que iniciativas desarrolladas en el contexto de la salud ocupacional en particular -y como política de salud pública en general- deben contar con la mejor metodología de diagnóstico posible, de modo de contribuir confiablemente a la evaluación de procesos de intervención en beneficio de la salud mental de trabajadores y trabajadoras de nuestro país. El SUSESO ISTAS extenso no es significativamente más caro que el breve.

Más allá de lo psicométrico, una reflexión de tipo teórico general nos lleva a decir que evaluar riesgos psicosociales desde la perspectiva de la salud ocupacional centrada en el riesgo o en lo negativo (Gil-Monte, 2012) obvia aspectos eventualmente positivos o salutogénicos que se producen en las personas como resultado de trabajar. Probablemente entonces, la generación y la aplicación en el futuro de enfoques integradores incluyendo aspectos de la psicología positiva por ejemplo, permitiría reconstruir desde el lenguaje la manera en que se conceptualiza el trabajo ya no solo como tripalium o como iatrogénico, sino como salutogénico o favorecedor de la salud y la realización de los trabajadores.

\section{Referencias}

Alvarado, R., Marchetti, N., Villalón, M., Hirmas, M. \& Pastorino, M. S. (2009). Adaptación y análisis psicométrico de un cuestionario para evaluar riesgos psicosociales en el trabajo en Chile: versión media del CoPsoQ. Revista Médica de Chile, 13, 7-16

Alvarado, R., Pérez-Franco, J., Saavedra, N., Fuentealba, C., Alarcón, A. \& Marchetti, N. (2012). Validación de un cuestionario para evaluar riesgos psicosociales en el ambiente laboral en Chile. Revista Médica de Chile, 140, 1154-1163.

Ansoleaga, E. (2015). Indicadores de salud mental asociados a riesgo psicosocial laboral en un hospital público. Revista Médica de Chile, $143,47-55$

Antúnez, Z. \& Vinet, E. (2012). Escalas de depresión, ansiedad y estrés (DASS-21): Validación de la Versión Abreviada en Estudiantes Universitarios Chilenos. Terapia Psicológica, 30, 49-55

Bravo, C. \& Nazar, G. (2015). Riesgo psicosocial en el trabajo y salud en conductores de locomoción colectiva urbana en Chile. Salud de los Trabajadores (Maracay), 23, 105-114

Ceballos, P., Paravic-Klijn, T., Burgos, M. \& Barriga, O. (2014). Validación de Escala Subjetiva de Carga Mental de Trabajo en funcionarios/as universitarios. Ciencia y Enfermería, 20, 73-82.

Ceballos-Vásquez, P., Rolo-González, G., Hérnandez-Fernaud, E., Díaz-Cabrera, D., Paravic-Klijn, T. \& Burgos-Moreno, M. (2015). Factores psicosociales y carga mental de trabajo: una realidad percibida por enfermeras/os en Unidades Críticas. Revista Latino-Americana de Enfermagem, 23, 315-322. doi: 10.1590/0104-1169.0044.2557

Cornejo, R. (2009). Condiciones de trabajo y bienestar/malestar docente en profesores de enseñanza media de Santiago de Chile. Educação \& Sociedade, 30 (107), 409-426 
Domínguez-Lara, S., \& Merino-Soto, C. (2015). ¿Por qué es importante reportar los intervalos de confianza del coeficiente alfa de Cronbach? Revista Latinoamericana de Ciencias Sociales, Niñez y Juventud, 13, 1326-1328

Domínguez-Lara, S., Merino-Soto, C. \& Navarro-Loli, J. (2016). Estimación paramétrica de la confiabilidad y diferencias confiables. Revista Médica de Chile, 144, 399-400.

Elosua, P. \& Zumbo, B. (2008). Coeficientes de fiabilidad para escalas de respuesta categórica ordenada. Psicothema, 20, 896-901

Garrido, P., Ansoleaga, E., Tomicic, A., Domínguez, C., Castillo, S., Lucero, C. \& Martínez, C. (2013). Afecciones de Salud Mental y el Proceso de Retorno al Trabajo: Una Revisión Sistemática. Ciencia \& trabajo, 15, 105-113.

Gil-Monte, P. (2012). Riesgos psicosociales en el trabajo y salud ocupacional. Revista Peruana de Medicina Experimental y Salud Pública, 29, 237-41

Gómez, P., Hernández, J. \& Mendez, M. D. (2014). Factores de Riesgo Psicosocial y Satisfacción Laboral en una Empresa Chilena del Área de la Minería. Ciencia \& Trabajo, 16, 9-16.

Guajardo, N., Ojeda, F., Achui, L. \& Larraguibel, M. (2015). Intervenciones terapéuticas para la conducta suicida en adolescentes. Revista Chilena de Psiquiatría, Neurología, Infancia y Adolescencia, 26, 145-155

Güilgüiruca, M., Meza, K., Góngora, R. \& Moya, C. (2015). Factores de riesgo psicosocial y estrés percibido en trabajadores de una empresa eléctrica en Chile. Medicina y Seguridad del Trabajo, 61, 57-67.

Instituto Sindical de Trabajo, Ambiente y Salud. (2003). Método ISTAS21 (CoPsoQ). Instrumento para la Prevención de Riesgos Psicosociales. Versión corta para pequeñas empresas y autoevaluación.

Kristensen, T. S., Hannerz, H., Høgh, A. \& Borg, V. (2005). The Copenhagen Psychosocial Questionnaire - a tool for the assessment and improvement of the psychosocial work environment. Scandinavian Journal Work, Environmental \& Health, 31, 438-449.

Lloret-Segura, S., Ferreres-Traver, A., Hernández-Baeza, A. \& TomásMarco, I. (2014). El análisis factorial exploratorio de los ítems: una guía práctica, revisada y actualizada Introducción Determinación de la adecuación del Análisis. Anales de Psicología, 30(3), 1151-1169.

Mendoza-Llanos, R. (2015). Insatisfacción laboral como predictor del ausentismo en un hospital público. Revista Médica de Chile, 143, 1028-1033

Ministerio de Salud. (2013). Protocolo de Vigilancia de Riesgos Psicosociales en el Trabajo. Gobierno de Chile

Minoletti, A. \& Zaccaria, A. (2005). Plan Nacional de Salud Mental en Chile: 10 años de experiencia. Revista Panamericana de Salud Pública, 18, 346-358.

Moncada, S., Llorens, C., Navarro, A. \& Kristensen, T. S. (2005). ISTAS21: Versión en lengua castellana del cuestionario psicosocial de Copenhague (COPSOQ). Archivos de Prevención de Riesgos Laborales, 8, 18-29.

Moncada, S., Utzet, M., Molinero, E., Llorens, C., Moreno, N., Galtés, A. \& Navarro, A. (2014). The Copenhagen Psychosocial Questionnaire II (CoPsoQ II) in Spain-A tool for Psychosocial Risk Assessment at the Workplace. American Journal of Industrial Medicine, 57, 97-107
Moyano-Díaz, E. \& Barría, R. (2006). Suicidio y Producto Interno Bruto (PIB) en Chile: Hacia un modelo predictivo. Revista Latinoamericana de Psicología, 38, 343-359.

OIT (2015). oIT: cada año mueren 2,3 millones de personas por accidentes o enfermedades laborales. Recuperado de http://noticias.universia.es/ empleo/noticia/2014/08/27/1110386/oit-cada-ano-mueren-2-3-millones-personas-accidentes-enfermedades-laborales.html

Organización Panamericana de la Salud. (2015). Plan de Acción sobre la salud de los trabajadores. $54^{\circ}$ Consejo Directivo. $67^{\mathrm{a}}$ Sesión del Comité Regional de la OMS para las Américas. 1 de octubre de 2015

Organización Mundial de la Salud. (2010). Ambientes de Trabajo Saludables: un modelo para la acción. Para empleadores, trabajadores, autoridades normativas y profesionales. Ediciones de la OMS: Ginebra

Román Hernández, J. (2006). El desencuentro entre la salud mental y la salud de los trabajadores. Revista Cubana de Salud Pública, 32.

Schermelleh-Engel, K., Moosbrugger, H. \& Müller, H. (2003). Evaluating the Fit of Structural Equation Models: Tests of Significance and Descriptive Goodness-of-Fit Measures. Methods of Psychological Research Online, 8, 23-74.

Silva, H., Lefio, A., Marchetti, N. \& Benoit, P. (2014). Riesgos Psicosociales en Conductores de Transporte de Carga y Pasajeros Urbanos e Interurbanos, y su Asociación con la Autopercepción de Salud y Siniestralidad Laboral. Ciencia \& Trabajo, 16, 67-74

Saavedra, N., Fuentealba, C. \& Pérez, J. M. (2009). SUSESO-ISTAS 21. Manual de uso Cuestionario de Evaluación de Riesgos Psicosociales en el Trabajo. Gobierno de Chile: Santiago.

Valdés, C. \& Errázuriz, P. (2012). Salud Mental en Chile: el pariente pobre del sistema de salud. Claves de Políticas Públicas. Instituto de Políticas Públicas: Universidad Diego Portales, 8, 1-10

Ximénez, C. \& García, A. (2005). Comparación de los métodos de estimación de máxima verosimilitud y mínimos cuadrados no ponderados en el análisis factorial confirmatorio mediante simulación Monte Carlo. Psicothema, 17, 528-535 